\title{
Tetrahydrobiopterin in biomedical research
}

\author{
Nenad Blau • Beat Thöny
}

Published online: 27 January 2009

(C) SSIEM and Springer 2009

Tetrahydrobiopterin $\left(\mathrm{BH}_{4}\right)$ is an endogenously synthesized cofactor, required for various enzyme activities and for some less well-defined functions. It is also required at the cellular level. The known enzymes that depend on $\mathrm{BH}_{4}$ are phenylalanine hydroxylase $(\mathrm{PAH})$, tyrosine hydroxylase, tryptophan hydroxylases 1 and 2, all three types of nitric oxide (NO) synthases (NOS), and glyceryl-ether monooxygenase (GEMO). At the cellular level, $\mathrm{BH}_{4}$ was found to be a growth or proliferation factor for Crithidia fasciculata and a control factor for infectivity of Leishmania major. In the nervous system, $\mathrm{BH}_{4}$ is a self-protecting factor for NO or a general neuroprotecting factor via the NOS pathway, and has dopamine-releasing function. The major controlling point of $\mathrm{BH}_{4}$ biosynthesis is GTP cyclohydrolase I (GTPCH) whose expression may be under control of cytokine induction, and at least in liver, GTPCH activity is inhibited by $\mathrm{BH}_{4}$ (and stimulated by phenylalanine) through the GTPCH feedback regulatory protein (GFRP). With regard to human disease, $\mathrm{BH}_{4}$ deficiency ('atypical PKU') due to autosomal recessive mutations in all cofactormetabolizing enzymes (except sepiapterin reductase) is described as a cause of hyperphenylalaninaemia (HPA). Under normal conditions the intracellular $\mathrm{BH}_{4}$ level is thought to play a pivotal role in not only the regulation of tyrosine and tryptophan hydroxylases, but also in the initial and rate-limiting steps in the biosynthesis of the catecholamines and serotonin.

\section{N. Blau $(\bowtie) \cdot$ B. Thöny}

Division of Clinical Chemistry and Biochemistry,

University Children's Hospital,

Zürich, Switzerland

e-mail: nenad.blau@kispi.uzh.ch
Thus, alterations in $\mathrm{BH}_{4}$ levels result in a disturbance of biogenic amine metabolism which has been implicated as an aetiological factor in a variety of neurological disorders. Mutations in the gene for GTPCH are closely related to Dopa-responsive dystonia (Segawa disease). This evidence strongly supports the importance of $\mathrm{BH}_{4}$ and dopaminergic transmission in dystonia. Alterations in $\mathrm{BH}_{4}$ metabolism have been observed in several neuropsychiatric diseases, such as Parkinson disease, familial dystonia, and endogenous depression, as well as in endothelial dysfunction, vitiligo, and more recently in pain perception. A subtype of PAH deficiency has been associated with $\mathrm{BH}_{4}$-responsiveness and mild or moderate phenylketonuria (PKU) due to specific mutations in the $P A H$ gene, offering potential pharmacological treatment with $\mathrm{BH}_{4}\left(\right.$ sapropterine; Kuvan $\left.{ }^{\mathrm{R}}\right)$. Biomedical research on $\mathrm{BH}_{4}$ expanded from the initial investigations in 'atypical PKU' patients to neurodegeneration, pain, vascular-endothelial, and epidermal dysfunction including diabetes, and other disciplines. Regarding treatment approaches, the field has been extended from $\mathrm{BH}_{4}$-cofactor and pharmachaperon treatment to enzyme replacement and experimental gene therapy.

The International Conference on Tetrahydrobiopterin, Phenylketonuria, and Nitric Oxide Synthase was organized in St. Moritz/Champér in Switzerland, on March 23-28, 2008, and hosted approximately 90 scientists from 15 different countries. More than 50 oral presentations were given and organized in various sessions including "Genetics and biochemistry of $\mathrm{BH}_{4}$ metabolism", "BH 4 metabolism and deficiency", " $\mathrm{BH}_{4}, \mathrm{GEMO}$, NOS and endothelial dysfunction", "PAH, PAH as misfolding disease, and $\mathrm{BH}_{4}$-responsive $\mathrm{PKU} / \mathrm{PAH}$ ", and "PKU (and new) 
treatment". The conference was sponsored by Biomarin Pharmaceutical Inc., Asubio Pharma Co., and Merck Serono SA. Generous sponsor support provided travelling awards for younger scientists and a special prize for the best presentation dedicated to the memory of Professor John Wood who died in 2008. In addition to the scientific highlights, one of the social emphases of the program was the traditional Pteridines Cup which took place on the slopes of Corviglia in the Alps surrounding St. Moritz (see picture). The next International Symposium on Pteridines and Folates will take place from June 7 to 12, 2009, in Jeju, Korea (http://www.biopku.org/bh42009/).

This issue of JIMD includes selected articles from the St. Moritz conference.

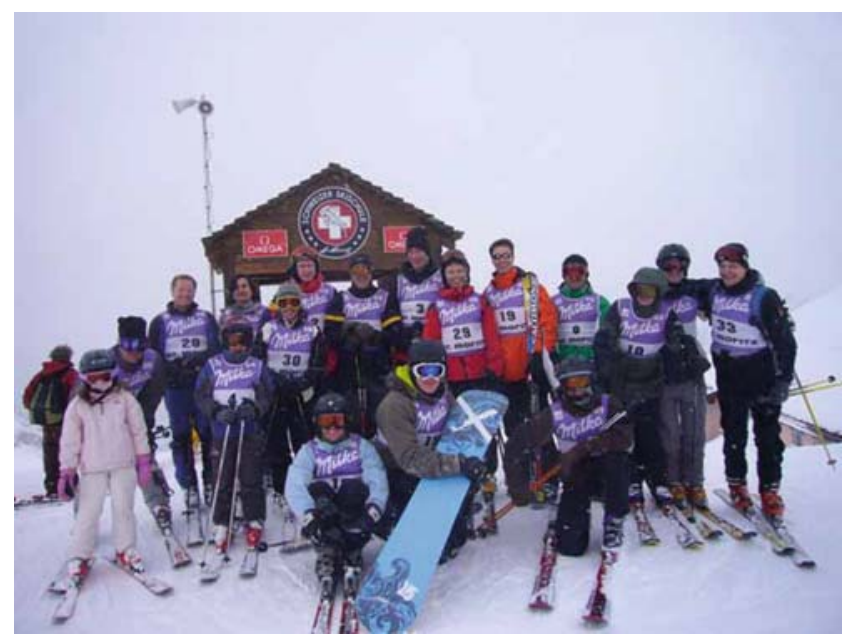

